\title{
Matéria Orgânica e Biomassa Microbiana em Plantios de Eucalipto no Cerrado Piauiense
}

\author{
Milton Marques Fernandes ${ }^{1}$, Marla Guedes Cordeiro Carvalho ${ }^{1}$, \\ Jader Magno Rodrigues de Araujo ${ }^{1}$, Francisco Rodolfo $\mathrm{Melo}^{2}$, \\ Carlos Alberto Silva ${ }^{3}$, Fabricio de Menezes Telo Sampaio², \\ Márcio Godofredo Rocha Lobato ${ }^{2}$ \\ ${ }^{1}$ Curso de Engenharia Florestal, Universidade Federal do Piauí - UFPI, Bom Jesus/PI, Brasil \\ ${ }^{2}$ Curso de Agronomia, Universidade Federal do Piauí - UFPI, Bom Jesus/PI, Brasil \\ ${ }^{3}$ Departamento de Solos - DS, Universidade Federal de Lavras - UFLA, Lavras/MG, Brasil
}

\begin{abstract}
RESUMO
Este trabalho objetivou determinar o efeito do plantio de eucalipto no carbono orgânico total, na biomassa microbiana e na matéria orgânica leve na região do Cerrado, no sul do Piauí. O estudo foi desenvolvido utilizando-se amostras de solos provenientes de povoamentos de Corymbia citriodora com quatro anos e Eucalyptus urophilla com cinco anos, e de uma área de cerrado preservada (testemunha). As práticas de manejo dos plantios de eucalipto promovem a oxidação da matéria orgânica leve, resultando em maior teor de carbono orgânico do solo em relação ao cerrado preservado. A biomassa microbiana do solo nos plantios de eucalipto apresentou pouca eficiência na manutenção do carbono em comparação ao cerrado preservado.
\end{abstract}

Palavras-chave: serapilheira, cerrado, florestas plantadas, carbono.

\section{Organic Matter and Microbial Biomass in Eucalyptus Plantations in the Cerrado of the State of Piaui}

\begin{abstract}
This study investigated the effect of eucalyptus plantations in the total organic carbon, microbial biomass and light organic matter from the cerrado in the south region of the State of Piaui. The study was carried out utilizing soil samples from four-year-old Corymbia citriodora and fiveyear-old Eucalyptus urophilla stands and a preserved cerrado area (control).The management practices of eucalyptus plantations promoted the oxidation of the light organic matter result in gin higher content of soil organic carbon in relation to the preserved cerrado area. The soil microbial biomass in eucalyptus plantations presented low efficiency in the maintenance of carbon compared to the preserved cerrado.
\end{abstract}

Keywords: litter, cerrado, forest plantations, carbon.

\section{INTRODUÇÃO}

O Cerrado piauiense ocupa uma área de quase 12 milhões de hectares, o que corresponde a $46 \%$ do Estado, 5,9\% do Cerrado brasileiro e 36,9\% do
Cerrado nordestino. Desse total, 70\% correspondem à área de cerrado; os $30 \%$ restantes compreendem a vegetação de transição com o bioma caatinga 
(Aguiar \& Monteiro, 2005). Nas últimas três décadas, tem-se observado expressivo aumento das atividades agrícolas e florestais em áreas do Cerrado, com intensa substituição da vegetação nativa por áreas cultivadas, especialmente para produção de alimentos, fibra e energia (Ferreira et al., 2007).

A substituição do cerrado por plantios de eucalipto podem resultar em decréscimo nos estoques de carbono (C), em função de incremento no processo erosivo, aceleração da decomposição da matéria orgânica do solo, redução no aporte de material vegetal ou diferenças na qualidade dos resíduos (Lima et al., 2008). Contudo, os resultados de diversos estudos - em que foi observado, por exemplo, o plantio do eucalipto - têm sido controversos, ou seja, em alguns casos, a substituição da vegetação nativa tem aumentado os estoques de C orgânico total (COT) (Rangel \& Silva, 2007), ao passo que, em outros trabalhos, esses estoques têm diminuído (Zinn et al., 2005).

Estudos têm demonstrado que determinados compartimentos da matéria orgânica do solo são capazes de detectar, mais rapidamente, as mudanças nos conteúdos de $\mathrm{C}$ no solo associadas ao manejo. As reduções nestes compartimentos são, de modo geral, maiores que as observadas, quando se considera apenas o conteúdo total de $\mathrm{C}$ do solo (Xavier et al., 2006). Numa escala crescente de sensibilidade, obtém-se, em primeira ordem, a biomassa microbiana do solo, bastante variável e sensível, considerada como compartimento ativo na dinâmica da matéria orgânica do solo (Lundquist et al., 1999); em seguida, representando uma medida de sensibilidade intermediária, obter-se-ia a matéria orgânica leve (Freixo et al., 2002).

O cerrado piauiense, nos últimos anos, vem aumentando sua área de plantio de eucalipto, em substituição à vegetação natural. Contudo, pouco se sabe sobre as alterações na matéria orgânica do solo ocasionadas pela substituição do cerrado pelo plantio de eucalipto. A partir do exposto, este trabalho objetivou determinar o efeito do plantio de eucalipto no carbono orgânico total, na biomassa microbiana e na matéria orgânica leve, na região de cerrado no sul do Piauí.

\section{MATERIAL E MÉTODOS}

O estudo foi realizado na Fazenda São Pedro, localizada na área da Serra do Quilombo, no município de Bom Jesus-PI $\left(9^{\circ} 4^{\prime} 26^{\prime \prime} \mathrm{S}\right.$ e $44^{\circ} 21^{\prime} 32^{\prime \prime} \mathrm{W}$, à altitude de $540 \mathrm{~m}$ ). O clima é do tipo Aw, conforme a classificação de Köppen, com clima quente e semiúmido e precipitação média anual de $900 \mathrm{~mm}$. O solo foi classificado como Latossolo Amarelo (Embrapa, 2006).

Foram selecionadas três áreas para o estudo, sendo uma área de plantio de eucalipto Corymbia citriodora, com cerca de 50 ha e aproximadamente quatro anos (EU4); plantio de Eucalyptus urophilla, com cerca de 55 ha e aproximadamente cinco anos (EU5), e uma área de referência de cerrado preservado (CP) (testemunha).

Em abril de 2010, coletaram-se, para cada área estudada, cinco amostras compostas, formadas por dez subamostras simples. As profundidades amostradas foram de $0-10 \mathrm{~cm}$ e $10-20 \mathrm{~cm}$ nas três áreas de estudo.

Após serem secas ao ar, as amostras deformadas foram destorroadas e passadas por peneira de $2 \mathrm{~mm}$, obtendo-se a terra fina seca ao ar (TFSA). No material, foi realizada a análise do carbono orgânico total, por oxidação da matéria orgânica via úmida com dicromato de potássio em meio sulfúrico (Embrapa, 1997).

Para determinação do carbono da biomassa microbiana do solo, foram utilizadas as mesmas amostras coletadas para determinação do carbono orgânico. As amostras foram acondicionadas em sacos plásticos e mantidas em refrigeração constante $\left(4{ }^{\circ} \mathrm{C}\right)$ por, no máximo, cinco dias. Um dia antes da realização das análises, as amostras foram retiradas do refrigerador e deixadas em temperatura ambiente por 24 horas. As amostras foram destorroadas, passadas em peneira de $2 \mathrm{~mm}$ de malha $\mathrm{e}$ homogeneizadas, retirando-se as raízes e os resíduos visíveis de plantas e animais do solo.

O carbono da biomassa microbiana foi determinado pelo método de irradiação-extração (Islam \& Weil, 1998; Mendonça \& Matos, 2005), utilizando-se forno de micro-ondas com potência de $900 \mathrm{~W}$ e frequência de $2.450 \mathrm{MHz}$. Após a irradiação, as amostras foram submetidas ao extrator sulfato de 
potássio $0,5 \mathrm{~mol} \mathrm{~L}^{-1}$; a determinação do carbono da biomassa microbiana foi feita por oxidação, com dicromato de potássio $0,066 \mathrm{~mol} \mathrm{~L}^{-1}$, e a titulação, com sulfato ferroso amoniacal $0,033 \mathrm{~mol} \mathrm{~L}^{-1}$. O cálculo do carbono da biomassa microbiana $\left(\mu \mathrm{g} \mathrm{kg}^{-1}\right.$ Cmicrobiano no solo) foi realizado por meio das expressões (Equação 1):

Cmicrobiano $=$ FC. $k c^{-1}$

em que: $\mathrm{FC}=\mathrm{CI}-\mathrm{CNI}$. Os elementos, nesta expressão, significam: $\mathrm{CI}=$ teor de carbono extraído do solo irradiado; $\mathrm{CNI}=$ teor de carbono extraído do solo não irradiado; Kc = fator de correção (valor de 0,33$)$.

A respiração basal foi avaliada pela quantificação do $\mathrm{CO}_{2}$ liberado; para essa determinação, a umidade das amostras foi corrigida para $70 \%$ da capacidade de campo. Em seguida, as amostras foram incubadas por 72 horas com solução de $\mathrm{NaOH} 0,05 \mathrm{~mol} \mathrm{~L}^{-1}$ e, posteriormente, tituladas com $\mathrm{HCl} 0,05 \mathrm{moL}^{-1}$, segundo Alef \& Nannipieri (1995).

A quantidade de $\mathrm{CO}_{2}$ liberada, cujo cálculo é apresentado em $\operatorname{mgC}-\mathrm{CO}_{2}\left(100 \mathrm{~cm}^{3}\right)^{-1}$ de solo, durante o intervalo de tempo utilizado no monitoramento da amostra, foi determinada a partir da seguinte Equação 2:

$\mathrm{C}-\mathrm{CO}_{2}(\mu \mathrm{g})=((\mathrm{Vo}-\mathrm{V}) \times 1,1)(\mathrm{PS} \times \mathrm{t})^{-1}$

em que: $P S$ = quantidade de solo em gramas; $\mathrm{t}$ = tempo de incubação em horas; $\mathrm{Vo}=$ volume do $\mathrm{HCl}$ para titulação do controle (média, se mais de controle for utilizado) $(\mathrm{mL}) ; \mathrm{V}=$ volume de HCL gasto na amostra $(\mathrm{mL})$.

A quantidade total de $\mathrm{C}-\mathrm{CO}_{2}$ produzida é igual ao somatório dos valores obtidos durante cada amostragem.

$\mathrm{O}$ quociente metabólico $\left(\mathrm{qCO}_{2}\right)$, que representa a quantidade de $\mathrm{CO}_{2}$ liberado por unidade de biomassa microbiana, foi obtido pela razão entre a respiração basal e o carbono da biomassa microbiana do solo (Anderson \& Domsch, 1978). Por sua vez, o quociente microbiano foi determinado pela razão entre o carbono da biomassa microbiana e o carbono orgânico do solo (Sparling, 1992).

Foram quantificados os teores de matéria orgânica leve (MOL) em água, segundo Anderson \& Ingram (1989). Para tanto, pesaram-se $50 \mathrm{~g}$ de terra fina seca ao ar (TFSA) em Becker de $250 \mathrm{~mL}$; em seguida, adicionaram-se $100 \mathrm{~mL}$ de solução de $\mathrm{NaOH} 0,1 \mathrm{~mol} \mathrm{~L}^{-1}$, sendo que este material permaneceu em repouso por uma noite. Decorrido o tempo, a suspensão foi agitada com bastão de vidro e todo o material passado por peneira de $0,25 \mathrm{~mm}$, eliminando-se toda a fração argila. Posteriormente, o material retido na peneira (MOL e areia) foi transferido novamente para o Becker, completando-se o volume com água. A porção sobrenadante foi passada por peneira de $0,25 \mathrm{~mm}$, tomando-se cuidado para separar a MOL da fração areia. Novamente, adicionou-se água ao Becker e, em seguida, empregou-se a agitação manual para suspender a MOL restante e verter este material vagarosamente em peneira de $0,25 \mathrm{~mm}$. Essa operação foi repetida até a completa remoção do material flotante. Após a separação, a MOL foi seca em estufa a $65^{\circ} \mathrm{C}$ até peso constante, sendo, posteriormente, pesada em balança de precisão. Além do peso seco, determinou-se o teor de carbono da MOL por meio de analisador automático (CHN analyzer Perkin Elmer 1420), segundo Yeomans \& Bremner (1988).

As áreas foram comparadas para cada variável e profundidade, individualmente. Foram realizadas análises de variância, aplicando-se o teste F. Para as variáveis cujo teste $\mathrm{F}$ foi significativo, compararam-se as médias estudadas, utilizando-se o teste de Tukey a $5 \%$ de probabilidade.

\section{RESULTADOS E DISCUSSÃO}

Em relação ao carbono orgânico total do solo nas áreas de plantio de eucalipto com quatro (EU4) e cinco (EU5) anos, nas profundidades de 0-10 cm e de 10-20 cm, não se verificou diferença estatística entre estas áreas; porém, houve diferença destas em relação à área de cerrado preservado nas duas profundidades. A área de cerrado foi a que apresentou menor teor de C orgânico, como observado na Tabela 1. Alcântara Neto et al. (2011) observaram maior valor de carbono orgânico total em área de cerrado em comparação a áreas de plantios de eucalipto com três e sete anos de idade, nas profundidades de 0-10 e $10-20 \mathrm{~cm}$. O padrão observado pelos autores pode ser atribuído à maior diversidade de plantas e à alta densidade de espécies 
Tabela 1. Carbono Orgânico Total das áreas de cerrado preservado (CP), plantios de eucalipto com quatro anos (EU4) e cinco anos (EU5).

Table 1. Total Organic Carbon of the cerrado areas preserved (CP), eucalyptus plantations in four years (EU4) and five years (EU5).

\begin{tabular}{lcc}
\multirow{2}{*}{ Áreas } & \multicolumn{2}{c}{ C Orgânico Total $\left(\mathbf{g} \cdot \mathbf{k g}^{-1}\right)$} \\
\cline { 2 - 3 } & $\mathbf{0 - 1 0} \mathbf{~ c m}$ & $\mathbf{1 0 - 2 0} \mathbf{~ c m ~}$ \\
\hline EU4 & $6,01^{\mathrm{a}}$ & $5,04^{\mathrm{a}}$ \\
EU5 & $5,26^{\mathrm{a}}$ & $4,26^{\mathrm{a}}$ \\
CP & $1,98^{\mathrm{b}}$ & $3,12^{\mathrm{b}}$ \\
\hline
\end{tabular}

Letras minúsculas diferentes indicam diferença estatística entre as áreas na profundidade de $0-10$ e $10-20 \mathrm{~cm}$ pelo teste de Tukey a $5 \%$.

arbóreas, o que resulta em maior aporte de resíduos vegetais ao solo.

A conversão do cerrado em plantios de eucalipto promoveu um incremento no teor de carbono orgânico total. Isto se deve, segundo Pulrolnik et al. (2009), ao fato de as plantações de eucalipto apresentarem taxas de crescimento superiores ao cerrado, depositando sobre o solo maior quantidade de folhas e resíduos finos. Pegoraro et al. (2011) explicam que o aumento da deposição de resíduos de eucalipto provenientes da parte aérea e das raízes pode contribuir substancialmente para o aumento do carbono orgânico total no solo e não somente para a substituição do carbono nativo do solo.

O carbono da biomassa microbiana (Cmic) apresentou maiores teores no cerrado preservado, cujos valores foram maiores que $90 \mathrm{mg}$ de carbono microbiano por grama de solo, sendo bastante superiores aos valores quantificados nas áreas sob cultivo de eucalipto, que foram inferiores a $45 \mu \mathrm{g}$ de carbono microbiano por grama de solo, não apresentando diferenças significativas entre si (Tabela 2).

O maior valor do carbono da biomassa microbiana no cerrado nativo é reflexo de uma situação bastante particular da comunidade microbiana do solo nesta área, que é estimulada pelo fornecimento contínuo de matéria orgânica com diferentes resistências à decomposição (Tabela 2). Além disso, a maior diversidade de espécies vegetais no cerrado preservado contribui para a deposição de substratos orgânicos oxidáveis com composição variada de serapilheira, favorecendo
Tabela 2. Carbono microbiano, respiração total acumulada, quociente microbiano e metabólico das áreas de cerrado preservado (CP), plantios de eucalipto com quatro anos (EU4) e cinco anos (EU5).

Table 2. Carbon microbial respiration cumulative total, and microbial metabolic quotient tof the cerrado areas preserved (CP), eucalyptus plantations in four years (EU4) and five years (EU5).

\begin{tabular}{lcc} 
Áreas & $\begin{array}{c}\text { C microbiano } \\
\left(\mu \mathbf{g . g}^{-1}\right)\end{array}$ & $\begin{array}{c}\text { Respiração total } \\
\text { acumulada } \\
\left(\mu \mathrm{g.g} \mathbf{~ d i a}^{-1}\right)\end{array}$ \\
\hline CP & $118,0^{\mathrm{a}}$ & $52,05^{\mathrm{a}}$ \\
\hline EU4 & $42,2^{\mathrm{b}}$ & $44,16^{\mathrm{b}}$ \\
\hline EU5 & $34,4^{\mathrm{b}}$ & $44,79^{\mathrm{b}}$ \\
\hline CP & Quociente microbiano & Quociente metabólico \\
\hline EU4 & $2,55^{\mathrm{a}}$ & $0,54^{\mathrm{a}}$ \\
\hline EU5 & $0,90^{\mathrm{b}}$ & $1,26^{\mathrm{b}}$ \\
\hline
\end{tabular}

Letras minúsculas diferentes indicam diferença estatística entre as áreas na profundidade de $0-10$ e $10-20 \mathrm{~cm}$ pelo teste de Tukey a $5 \%$.

uma maior diversidade de compostos orgânicos depositados na rizosfera; esse fato contribui para a sobrevivência e o crescimento dos diferentes grupos de microrganismos do solo (D’Andréa et al., 2002). Nos plantios de eucalipto, a presença de substâncias com atividade antimicrobiana encontradas na serapilheira dificulta a sobrevivência e o crescimento dos diferentes grupos de microrganismos do solo (Alvarenga et al., 1999).

A liberação de $\mathrm{C}-\mathrm{CO}_{2}$ decorrente da atividade microbiana do solo foi maior no $\mathrm{CP}$, quando comparada à dos plantios de eucalipto. Contudo, não foram observadas diferenças significativas entre os tratamentos EU4 e EU5, sendo esse padrão similar ao verificado para a biomassa microbiana do solo (Tabela 2). Nos plantios de eucalipto, só existe uma única fonte de material formador de serapilheira, com composição química de difícil decomposição pela biomassa microbiana. De acordo com Costa et al. (2005), a serapilheira dos eucaliptos apresenta muita lignina, o que dificulta a penetração de microorganismos decompositores por meio da parede celular, contribuindo, assim, para pequena decomposição.

Em relação ao quociente metabólico, verifica-se que não houve diferença significativa entre as médias dos plantios de EU4 e EU5. Note-se que os 
valores apresentados para os plantios de eucalipto de quatro e cinco anos, respectivamente, 1,26 e $1,48 \mu \mathrm{g} \mathrm{g}^{-1}$, são mais do que o dobro do observado no cerrado preservado, que apresentou $0,54 \mu \mathrm{g} \mathrm{g}^{-1}$ (Tabela 2). Segundo Tótola e Chaer (2002), quanto menor o quociente metabólico, maior é a economia na utilização de energia, além de haver, ainda, um ambiente mais estável ou mais próximo do seu estado de equilíbrio. O quociente metabólico, à medida que determinada população microbiana se torna mais eficiente, há uma menor perda de $\mathrm{C}$ como $\mathrm{CO}_{2}$ pela respiração e maior proporção de $\mathrm{C}$ seria incorporada ao tecido microbiano (Gama-Rodrigues, 2005).

Para o quociente microbiano, o menor valor foi obtido na área de plantio de eucalipto com quatro anos (EU4), com $0,90 \mu \mathrm{g} \mathrm{g}^{-1}$, indicando que nesse sistema houve menor eficiência dos microrganismos em imobilizar carbono quando comparado com o cerrado preservado (CP); no entanto, o plantio de eucalipto com cinco anos (EU5), com maior tempo de plantio, apresentou quociente microbiano similar ao cerrado preservado (Tabela 2). A presença de maior quociente microbiano está associada, provavelmente, ao equilíbrio existente no sistema por conta da estreita e harmônica integração da cobertura vegetal com o sistema físico, químico e biológico do solo, por meio dos processos essenciais, como a ciclagem de nutrientes, resultante da formação e da decomposição da matéria orgânica (Leite et al., 2003).

Segundo Gama-Rodrigues e Gama-Rodrigues (2008), em solos com adição de serapilheira de baixa qualidade nutricional, a biomassa microbiana encontra-se sob estresse e é incapaz de utilizar totalmente o C orgânico; então, nesse caso, o quociente microbiano tende a diminuir. Assim, o menor valor observado no solo sob o plantio de eucalipto com quatro anos indica uma provável condição de estresse para a população microbiana, decorrente principalmente da redução da quantidade e da qualidade de substrato orgânico na formação de serapilheira (Tabela 2).

Observou-se que, nas profundidades de 0-10 e 10-20 cm, nos plantios de eucalipto com quatro e cinco anos, a quantidade de matéria orgânica leve (MOL) foi estatisticamente menor do que na área de cerrado preservado (Tabela 3). A diferença de um
Tabela 3. Carbono da matéria orgânica leve das áreas de cerrado preservado (CP), plantios de eucalipto com quatro anos (EU4) e cinco anos (EU5).

Table 3. Carbon light organic matter preserved cerrado areas (CP), eucalyptus plantations in four years (EU4) and five years (EU5).

\begin{tabular}{crc} 
& \multicolumn{2}{c}{$\left(\mathbf{g} \cdot \mathbf{k g}^{-1}\right)$} \\
\cline { 2 - 3 } Áreas & $\mathbf{0 - 1 0} \mathbf{~ c m}$ & $\mathbf{1 0 - 2 0} \mathbf{~ c m}$ \\
\hline EU4 & $59,8^{\mathrm{a}}$ & $32,9^{\mathrm{a}}$ \\
EU5 & $57,0^{\mathrm{a}}$ & $33,8^{\mathrm{a}}$ \\
CP & $106,2^{\mathrm{b}}$ & $56,5^{\mathrm{b}}$ \\
\hline
\end{tabular}

Letras minúsculas diferentes indicam diferença estatística entre as áreas na profundidade de $0-10$ e $10-20 \mathrm{~cm}$ pelo teste de Tukey a $5 \%$.

ano entre os plantios EU4 e EU5 não estabeleceu diferenças significativas nos valores de carbono da matéria orgânica leve. Observa-se que, nos plantios de eucalipto com quatro (EU4) e cinco anos (EU5), não foi aportada serapilheira suficiente para manter o carbono da matéria orgânica leve semelhante à do cerrado preservado. Lima et al. (2008) observaram que, mesmo transcorridos 30 anos de plantio de eucalipto na região do Vale do Rio Doce, em Minas Gerais, não se apresentou um teor de matéria orgânica leve maior do que a da área de floresta.

De acordo com Pulrolnik et al. (2009), se o plantio de eucalipto aporta menor quantidade de serapilheira que o cerrado, ocorre uma redução no carbono da matéria orgânica leve do solo. Segundo Alcântara Neto et al. (2011), o revolvimento do solo para o plantio de eucalipto na região do cerrado causa a quebra dos agregados e expõe a matéria orgânica fisicamente protegida à atuação microbiana, aumentando as condições de aeração; assim, esse quadro resultante contribui para a rápida decomposição, especialmente daquelas formas mais prontamente disponíveis ou lábeis, como o $\mathrm{C}$ da matéria orgânica leve do solo. De acordo com Loss et al. (2010), o revolvimento do solo desfavorece a manutenção da matéria orgânica leve do solo.

\section{CONCLUSÕES}

As práticas de manejo dos plantios de eucalipto promovem a oxidação da matéria orgânica leve, resultando em maior teor de carbono orgânico do solo em relação ao cerrado preservado. 
A biomassa microbiana do solo nos plantios de eucalipto apresentou pouca eficiência na manutenção do carbono em comparação ao cerrado preservado.

\section{STATUS DA SUBMISSÃO}

Recebido: 07/03/2012

Aceito: 01/10/2012

Publicado: 31/12/2012

\section{AUTOR(ES) PARA CORRESPONDÊNCIA}

\section{Milton Marques Fernandes}

Curso de Engenharia Florestal,

Universidade Federal do Piauí - UFPI,

Campus Professora Cinobelina Elvas - CPCE,

Rod. BR 135, Km 3, Planalto Horizonte,

CEP 64900-000, Bom Jesus, PI, Brasil

e-mail: miltonmf@gmail.com

\section{REFERÊNCIAS}

Aguiar TJA, Monteiro MSL. Modelo agrícola e desenvolvimento sustentável: A ocupação do cerrado piauiense. Ambiente e Sociedade 2005; 8(2): 1-18. http:// dx.doi.org/10.1590/S1414-753X2005000200009

Alcântara Neto F, Leite LFC, Arnhold E, Maciel GA, Carneiro FV. Compartimentos de carbono em Latossolo Vermelho sob cultivo de eucalipto e fitofisionomias de cerrado. Revista Brasileira de Ciência do Solo 2011; 35: 849-856. http://dx.doi.org/10.1590/ S0100-06832011000300019

Alef K, Nannipieri P. Methods in applied soil microbiology and biochemistry. London: Academic Press; 1995. 237 p.

Alvarenga MIN, Siqueira JO, Davide AC. Teor de carbono, biomassa microbiana, agregação e micorriza em solos de Cerrado com diferentes usos. Ciência Agrotécnica 1999; 23(3): 617-625.

Anderson JPE, Domsch KH. A physiological method for the quantitative measurement of microbial biomass in soil. Soil Biology \& Biochemistry 1978; 10: 215-221. http://dx.doi.org/10.1016/0038-0717(78)90099-8

Anderson JM, Ingram JSI. Tropical soil biology and fertility: A handbook of methods. Wallingford: CAB International; 1989. 305 p.

Costa GS, Gama-Rodrigues AC, Cunha GM. Decomposição e liberação de nutrientes da serapilheira foliar em povoamentos de Eucalyptus grandis no Norte Fluminense. Revista Árvore 2005; 29(9): 563-570. http:// dx.doi.org/10.1590/S0100-67622005000400008
D’Andréa AF, Silva MLN, Curi N, Siqueira JO, Carneiro MAC. Atributos biológicos indicadores da qualidade do solo em sistemas de manejo na região do cerrado no sul do Estado de Goiás. Revista Brasileira de Ciência do Solo 2002; 26: 913-923.

Embrapa. Centro Nacional de Pesquisa de Solos. Manual de métodos de análise de solo. 2. ed. Rio de Janeiro: Embrapa; 1997. 280 p.

Embrapa. Centro Nacional de Pesquisa de Solos. Sistema brasileiro de classificação de solos. 2. ed. Rio de Janeiro: Embrapa; 2006. 306 p.

Ferreira EAB, Resck DVS, Gomes AC, Ramos MLG. Dinâmica do carbono da biomassa microbiana em cinco épocas do ano em diferentes sistemas de manejo do solo no cerrado. Revista Brasileira de Ciência do Solo 2007; 31(6): 1625-1635, 2007. http://dx.doi. org/10.1590/S0100-06832007000600038

Freixo AA, Machado PLOA, Guimarães CM, Silva CA, Fadigas FS. Estoques de carbono e nitrogênio e distribuição de frações orgânicas de Latossolo do cerrado sob diferentes sistemas de cultivo. Revista Brasileira de Ciência do Solo 2002; 26(1): 425-434.

Gama-Rodrigues EF. Nitrogênio, carbono e atividade da biomassa microbiana do solo em plantações de eucalipto. Revista Brasileira de Ciência do Solo 2005; 29: 893-901. http://dx.doi.org/10.1590/S010006832005000600007

Gama-Rodrigues EF, Gama-Rodrigues AC, Paulino GM, Franco AA. Atributos químicos e microbianos de solos sob diferentes coberturas vegetais no norte do Estado do Rio de Janeiro. Revista Brasileira de Ciência do Solo 2008; 32: 1521-1530. http://dx.doi.org/10.1590/ S0100-06832008000400016

Islam KR, Weil RR. Microwave irradiation of soil for routine measurement of microbial biomass carbon. Biology and Fertility of Soils 1998; 27(4): 408-416. http:// dx.doi.org/10.1007/s003740050451

Leite LFC, Mendonça ES, Machado PLOA, Matos ES. Total C and N storage and organic C pools of a RedYellow Podzolic under conventional and no tillage at the Atlantic Forest Zone, Southeastern Brazil. Australian Journal of Soil Research 2003; 41(4): 717-730. http:// dx.doi.org/10.1071/SR02037

Lima AMN, Silva IR, Neves JCL, Novais RF, Barros NF, Mendonça ES. Frações da matéria orgânica do solo após três décadas de cultivo de eucalipto no Vale do Rio Doce-MG. Revista Brasileira de Ciência do Solo, 2008, 32(2): 1053-1063. http://dx.doi.org/10.1590/ S0100-06832008000300014

Loss A, Moraes AGL, Pereira MG, Silva EMR, Anjos LHC. Carbono, matéria orgânica leve e frações oxidáveis do carbono orgânico sob diferentes sistemas de produção orgânica. Comunicata Scientiae 2010; 1(1): 57-64. 
Lundquist EJ, Jackson LE, Scow KM, Hsu C. Changes in microbial biomass and community composition, and soil carbon and nitrogen pools after incorporation of rye into three California agricultural soils. Soil Biology Biochemistry 1999; 31(2): 221-236. http://dx.doi. org/10.1016/S0038-0717(98)00093-5

Mendonça ES, Matos ES. Matéria orgânica do solo; métodos de análises. Viçosa: UFV; 2005. 107 p.

Pegoraro RF, Silva IR, Novais RF, Barros NF, Fonseca $S$, Dambroz CS. Estoques de carbono e nitrogênio nas frações da matéria orgânica em Argissolo sob eucalipto e pastagem. Ciência Florestal 2011; 21(2): 261-273.

Pulrolnik K, Barros NF, Silva IR, Novais RF, Brandão NICB. Estoques de carbono e nitrogênio em frações lábeis e estáveis da matéria orgânica de solos sob eucalipto, pastagem e Cerrado no vale do Jequitinhonha - MG. Revista Brasileira de Ciência do Solo 2009; 33(3): 1125-1136. http://dx.doi.org/10.1590/ S0100-06832009000500006

Rangel OJP, Silva CA. Estoques de carbono e nitrogênio e frações orgânicas de Latossolo submetido a diferentes sistemas de uso e manejo. Revista Brasileira Ciência do Solo 2007; 31(2): 1609-1623.

Sparling GP. Ratio of microbial biomass carbon to soil organic carbon as a sensitive indicator of changes in soil organic matter. Australian Journal Soil Resources 1992; 30(4): 195-207. http://dx.doi. org/10.1071/SR9920195

Tótola MR, Chaer GM. Microrganismos e processos microbiológicos como indicadores da qualidade do solo. In: Alvarez Venegas VH, Schaefer CEGR, Barros NF, Mello JWV, Costa LM, editor. Tópicos em ciência do solo. Viçosa: Sociedade Brasileira de Ciência do Solo; 2002.

Xavier FAZ, Maia SMF, Oliveira TS, Mendonça ES. Biomassa microbiana e matéria orgânica leve em solos sob sistemas agrícolas, orgânico e convencional na Chapada da Ibiapaba - CE. Revista Brasileira de Ciência do Solo, 2006; 30(1): 247-258. http://dx.doi.org/10.1590/ S0100-06832006000200006

Yeomans JC, Bremner JM. A rapid and precise method for routine determination of organic carbon in soil. Communications in Soil Science and Plant Analysis 1988; 19(1): 1467-1476. http://dx.doi. org/10.1080/00103628809368027

Zinn YL, Lal R, Resck DVS. Changes in soil organic carbon stocks under agriculture in Brazil. Soil Tillage Resources 2005; 84(2): 28-40. http://dx.doi. org/10.1016/j.still.2004.08.007 